\title{
Impact of inter-facility transport on maternal mortality in the Free State Province
}

\author{
M G Schoon, ${ }^{1,2}$ MMed (O\&G), PhD \\ ${ }^{1}$ Free State Department of Health, South Africa \\ ${ }^{2}$ Faculty of Health Sciences, Free State University, South Africa
}

Corresponding author: M G Schoon (schoonm@fshealth.gov.za)

\begin{abstract}
Aim. In December 2011, having identified inter-facility transport as a problem in the maternity service, the Free State Department of Health procured and issued 48 vehicles including 18 dedicated to maternity care. Subsequently, a sustained reduction in mortality was observed. We probed the role of inter-facility transport in effecting this reduction in mortality.

Methods. A before-after analysis was performed of data from 2 separate databases, including the district health information system and the emergency medical and rescue services call-centre database. Data were compared for a 12-month prior- and 10-month post-intervention period using descriptive and correlation statistics.

Results. The maternal mortality decreased from 279/100 000 live births during 2011 to 152/100 000 live births during 2012. The mean dispatch interval decreased from 32.01 to 22.47 minutes. The number of vehicles dispatched within 1 hour increased from $84.2 \%$ to $90.7 \%$ $(p<0.0001)$. Monthly mean dispatch interval curves closely mirrored the maternal mortality curve.

Conclusion. Effective and prompt inter-facility transport of patients with pregnancy complications to an appropriate facility resulted in a reduction of maternal mortality. Health authorities should prioritise funding for inter-facility vehicles for maternity services to ensure prompt access of pregnant women to centres with skills available to manage obstetric emergencies.

S Afr Med J 2013;103(8):534-537. DOI:10.7196/SAMJ.6828
\end{abstract}

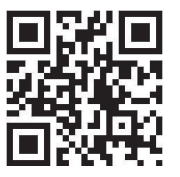

Maternal mortality in the Free State Province ranks among the highest in South Africa (SA). Various strategies are being implemented to reduce the mortality rates within the province. Reports of adverse events identified transport as a contributing factor.

It has been documented that access to emergency obstetric care and reduced transport time to such centres for treatment reduce deaths. ${ }^{[1,2]}$ The 'Three Delays Model' proposed by Thaddeus and Maine ${ }^{[3]}$ attempts to describe where gaps occur in obstetric management: (i) delay in seeking help on the part of individual patients; (ii) delay in reaching a suitable healthcare facility; and (iii) delay in receiving adequate care at that facility. The confidential enquiries into maternal deaths have identified transport issues as a factor relating to deaths. ${ }^{[4]}$ When dealing with maternal mortality, deaths can be avoided by timely and adequate treatment ${ }^{[5]}$ and programmes that reduce barriers to comprehensive emergency obstetric care can substantially reduce deaths associated with obstetric emergencies. ${ }^{[1]}$

In the Free State Province of SA, antenatal care is provided at all primary healthcare (PHC) clinics. While $90 \%$ of the community has access to these sites, giving birth before reaching these remains a problem. Confinements occur in formal labour wards, but not all hospitals have the staffing profile to provide a 24-hour comprehensive emergency obstetric care service. In dealing with the second delay referred to above, the largest challenge has been to ensure that patients entering PHC clinics and sub-optimally functioning hospitals are transported to a centre with appropriate staffing and skills to provide comprehensive obstetric emergency care. To the frustration of maternity staff, there have been lengthy waiting periods for an ambulance to be dispatched to transfer a pregnant woman in labour to an appropriate facility. Inter-facility transport was viewed as deserving a lesser priority in the view of emergency medical and rescue services (EMRS) professionals whose general belief is that 'any patient in a health facility is better off than a patient at home. Yet, organised ambulance services have been identified as integral to the success of national programmes aimed at reducing maternal mortality. ${ }^{[6]}$ In response, the Department of Health (DoH) decided to provide 48 ambulances for inter-facility transport; 18 were earmarked for dedicated inter-facility maternity transfers. These vehicles were distributed to facilities during December 2011. Various other interventions were conducted within the province in an attempt to reduce mortality. These have included training on the management of obstetric emergencies, improvement of antenatal care and review of service planning.

The purpose of this study was to explore the impact of dedicated obstetric inter-facility transport on maternal mortality.

\section{Methods}

I performed a retrospective analysis of the effect of inter-facility transport on maternity maternal mortality over a period of 12 months before, and 10 months after, the provision of 18 staffed ambulances dedicated to maternal inter-facility transport.

Maternal mortality data were collected from the provincial District Health Information System (DHIS). This included monthly data on the number of deliveries, live births, stillbirths and neonatal deaths. Maternal deaths were collected from the provincial maternal death notification register. The period of data collection was from 1 January 2011 to 30 October 2012.

From the EMRS database, dispatch data were downloaded for the same period onto a Microsoft Excel worksheet. The incident types, registered call date and time, dispatch date and time, vehicles, and comments were selected for the category 'inter-facility transportmaternity'. The time of each call was calculated and fields were added to identify the calls that were dispatched within 15 minutes and 1 hour. Statistical data analysis was performed with Epi Info 7 and StatCalc, and included means, median and modes with standard deviations stratified per month for the entire period. 
Table 1. Comparative data before and after the issue of inter-facility vehicles

\begin{tabular}{|c|c|c|}
\hline & $\begin{array}{l}2011 \\
\text { (January - December) }\end{array}$ & $\begin{array}{l}2012 \\
\text { (January - October) }\end{array}$ \\
\hline Delivery in facility, $n$ & 46979 & 40462 \\
\hline Live birth in facility, $n$ & 46170 & 39924 \\
\hline $\mathrm{BBA}$ rate, ${ }^{\dagger} \%$ & 9.29 & 9.05 \\
\hline Maternal death in facility, $n$ & 129 & 61 \\
\hline MMR/100 000 live births & 279.4 & 152.8 \\
\hline Inter-facility transfers registered, $n$ & 9341 & 9413 \\
\hline Vehicles dispatched after call registered, $n$ & 9186 & 8966 \\
\hline Inter-facility transfer rate, ${ }^{*} \%$ & 19.6 & 22.2 \\
\hline Vehicles dispatched within 1 hour, $n$ & 7738 & 8132 \\
\hline Vehicles dispatched within 1 hour, ${ }^{*} \%$ & 84.2 & 90.7 \\
\hline $\begin{array}{l}\text { Register dispatch interval (min), } \\
\text { average } \pm \text { SD (median) }\end{array}$ & $32.01 \pm 75.56(10.16)$ & $22.47 \pm 77.67(6.65)$ \\
\hline \multicolumn{3}{|c|}{$\begin{array}{l}\text { BBA = birth before arrival; } \mathrm{MMR}=\text { maternal mortality rate; } \mathrm{SD}=\text { standard deviation. } \\
{ }^{*} p<0.001 \\
{ }_{p} p 0.1\end{array}$} \\
\hline
\end{tabular}

Table 2. Event description at closure of call

\begin{tabular}{lll}
\hline & $\mathbf{2 0 1 1}$ & $\mathbf{2 0 1 2}$ \\
\hline Airlifted & - & $\boldsymbol{n}$ \\
Cancelled by caller & 253 & 213 \\
Died before transfer & 7 & 9 \\
Died during transport & 1 & - \\
Double booking & 46 & 64 \\
Hoax call & 2 & - \\
No escort found & 4 & 3 \\
No patient found & 43 & 24 \\
No response from caller & - & 2 \\
Out of area & 2 & 5 \\
Patient delivered & 11 & 11 \\
Patient not ready & 4 & 2 \\
Patient removed by another ambulance & 23 & 10 \\
Patient transported privately & 20 & 8 \\
Reason unknown & 1 & - \\
Private ambulance service & 1 & 8 \\
Private transport used & 40 & 13 \\
Transfer to another district & - & 21 \\
Total number of events, $N$ & 458 & 394 \\
& &
\end{tabular}

\section{Results}

Since early 2012, a marked sustained drop in maternal mortality occurred (Fig. 1). Of the 112901 inter-facility calls, 18754 logged calls were dedicated to maternity transfers and 17318 were dispatched. Table 1 summarises the maternal data and interfacility calls. Comments regarding problems with calls are listed in Table 2. This includes those where vehicles were not dispatched or, live births) in 2011 to $61(152 / 100000$ live births) in the period of January to October 2012 ( $p=0.000076$; risk ratio (RR) 0.54 ; $95 \%$ confidence interval (CI) 0.40 - 0.74). During 2011, a total of 9186 vehicles were dispatched with an average call register-dispatch time of 32.01 minutes, with a total of $7738(84.2 \%)$ vehicles having been dispatched within 1 hour. In 2012, 8966 vehicles were dispatched, with a mean dispatch time of 22.47 minutes; 8132 (90.7\%) were dispatched within 1 hour.

The correlation between mean call register-dispatch time and maternal deaths $\left(r^{2}=0.5913 ; p=0.000034\right)$ and the proportion of dispatched vehicles within 1 hour $\left(r^{2}=0.5729 ; p=0.000051\right)$ show a moderate correlation with reduction in mortality associated with shorter dispatch times and higher proportion dispatched within 1 hour.

The proportion of vehicles dispatched within 1 hour increased from $84.2 \%$ in 2011 to $90.7 \%$ in 2012 ( $p<0.0001$ ) (odd's ratio (OR) 1.82; 95\% CI 1.67 - 1.99). Following the issuing of vehicles, a clear sustained change in the mortality associated with a sustained improvement in the $<1$ hour dispatch rate could be demonstrated (Fig. 2). More remarkable is the association between the fall in mean dispatch time and the decline in maternal mortality (Fig. 3).

The proportion of transfers during dayshift did not significantly differ between the 2 periods $(72.6 \%$ in $2011 ; 72.5 \%$ in 2012; $p=0.9$ ). The distribution of the calls between urban and rural areas was also similar during the 2 periods (for urban areas: $66.48 \%$ in $2011,65.2 \%$ in 2012; $p=0.07$ ).

An analysis of deaths revealed that 67 (54\% of notified deaths) occurred after referral in 2011 compared with 24 (46.2\%) in 2012. This reflects a reduction of mortality among referred patients from 729.3/100 000 transfers in 2011 to $267 / 100000$ transfers in 2012 ( $p=0.000011$; RR 0.36; 95\% CI 0.23 - 0.58).

During 2011, the proportion of calls dispatched within 1 hour was higher in the rural areas $(86.4 \%)$ compared with the urban areas $(83.1 \%$; $p=0.000036)$ while during 2012 , the proportions were similar in rural and urban areas (90.4\% and $90.8 \%$, respectively; $p=0.5413$ ). Calls dispatched within 1 hour improved for both the dayshifts, from $82.6 \%$ in 2011 to $89.1 \%$ in $2012(p<0.0001)$ and the nightshifts from $88.36 \%$ in 2011 to $92.7 \%$ in $2012(p<0.0001)$. The mean dispatch intervals in 2011 for urban and rural transfers of 33.59 and 28.8 minutes improved to 23.27 and 20.97 minutes, respectively.

There were no significant changes in the born-before-arrival (BBA) rates $(9.29 \%$ v. $9.05 \%, p=0.2282$ ). There was, however, a significant correlation between the monthly BBA rate and perinatal mortality $\left(r^{2}=1\right)$. 


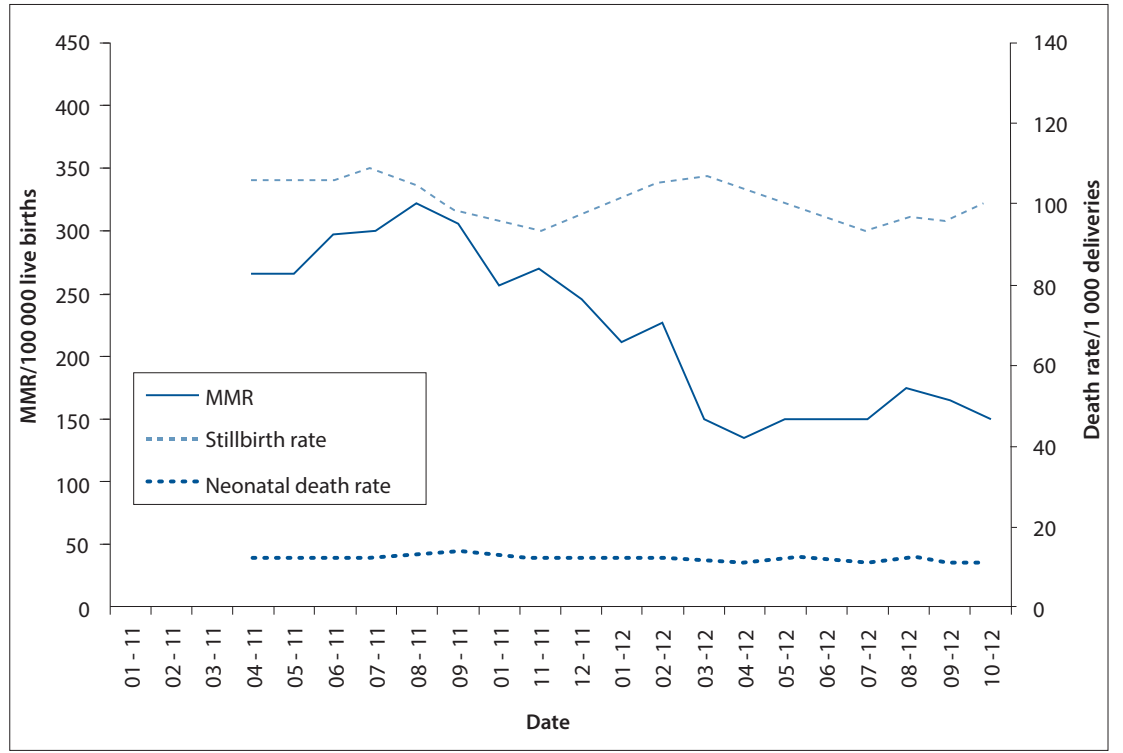

Fig. 1. Maternity-related mortalities. $M M R=$ maternal mortality rate.

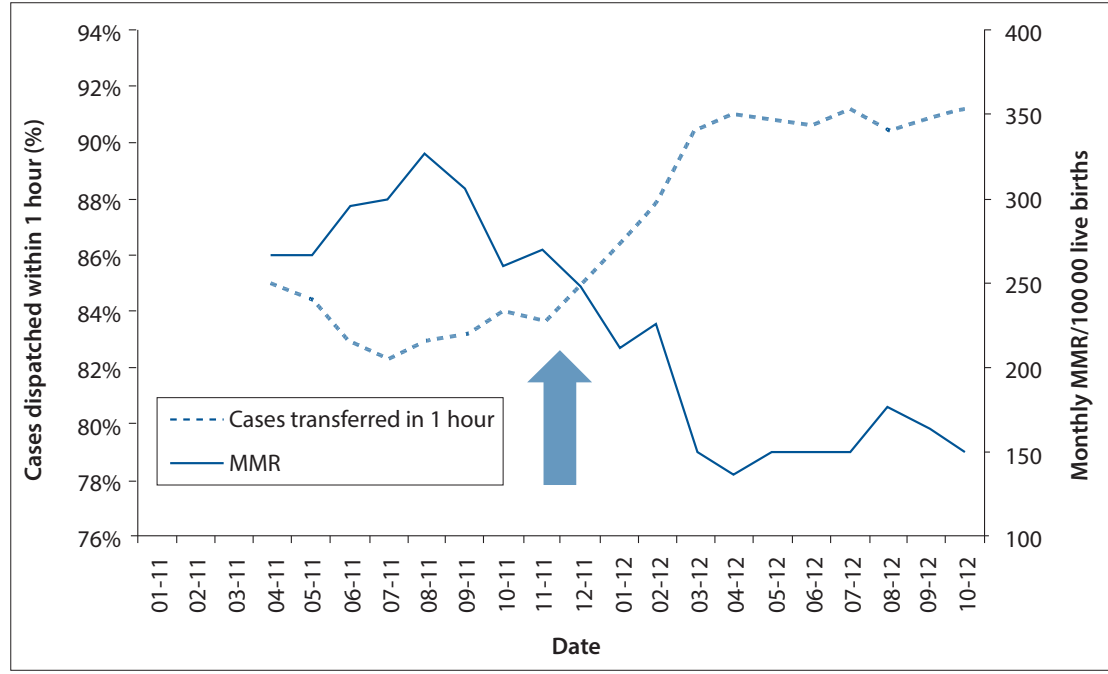

Fig. 2. Relationship between inter-facility vehicles dispatched within 1 hour and maternal mortality. The arrow indicates when vehicles were issued.

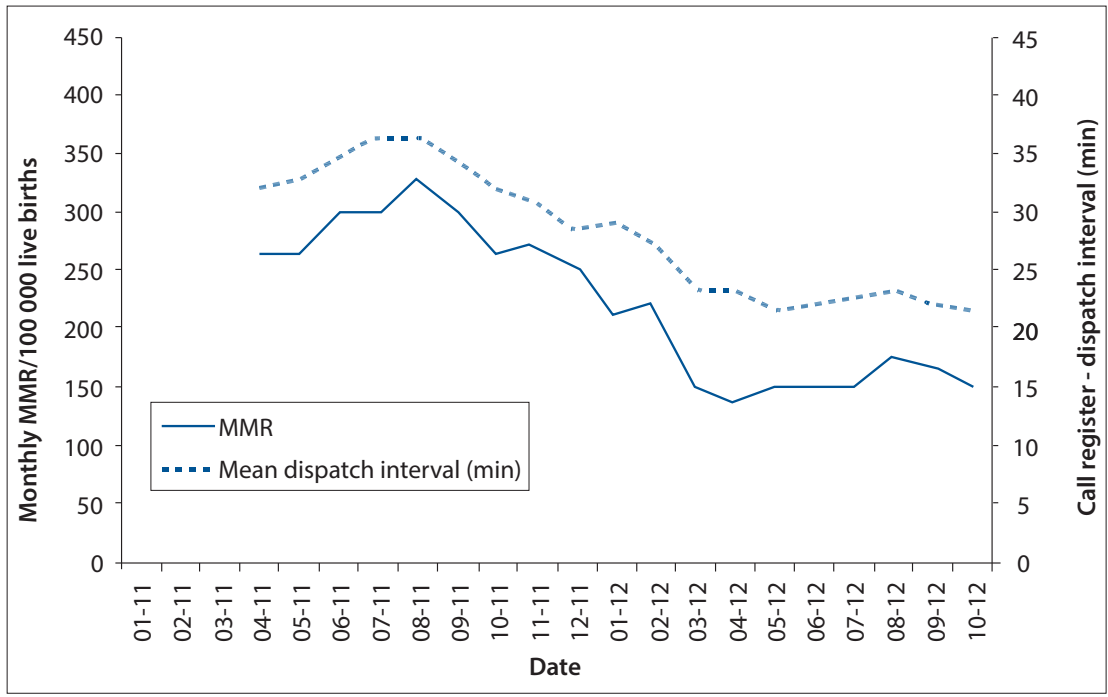

Fig. 3. Mean dispatch interval ( $\mathrm{min}$ ) and maternal mortality rate (MMR).

\section{Discussion}

As a resource-challenged province, various programmatic activities have been executed by the $\mathrm{DoH}$ in an attempt to reduce the high maternal mortality rates. Among these strategies, the Free State DoH prioritised staffed maternity inter-facility transport in December 2011 in response to the recorded adverse events that indicated that interfacility transport related factors contributed to deaths. ${ }^{[7]}$ An indirect correlation between maternal mortality and vehicles dispatched would prove that improvement occurred as a result of this transport-related intervention.

The maternal mortality, stillbirths and neonatal mortality rates over time (Fig. 1) demonstrate a profound decrease in maternal mortality since December 2011, with a lesser trend to reduction in stillbirths. When the mortality rates of patients referred were calculated, this decline in mortality becomes even more profound, emphasising the importance of the inter-facility transport intervention. There was, however, no change in the neonatal mortality over time.

The correlation between mean call register-dispatch time and maternal deaths $\left(r^{2}=0.5913 ; p=0.000034\right)$ and proportion of dispatched vehicles within 1 hour $\left(r^{2}=0.5729\right.$; $p=0.000051$ ) showed a clear reduction in mortality associated with shorter dispatch times and higher proportion dispatched within 1 hour (Figs 2 and 3, respectively).

The proportion of vehicles dispatched within 1 hour increased from $84.2 \%$ in 2011 to $90.7 \%$ in 2012 ( $p<0.0001$; OR 1.82; 95\% CI 1.67 - 1.99). Following the issuing of vehicles a clear sustained change in the mortality associated with a sustained improvement in the $<1$-hour dispatch rate could be demonstrated (Fig. 2). More interesting is the association between the drop in mean dispatch time with the decline in maternal mortality (Fig. 3).

It is important to note that this transport intervention supplied vehicles with basic emergency personnel aimed at providing 24-hour cover. This intervention can be regarded as providing improved wheels, but not skills, given that emergency personnel received an average of 6 weeks of basic obstetric first aid training only. This basic training did, however, avoid depleting facilities and especially small facilities of their skilled midwife/midwives during the period of transfer.

Serious challenges were experienced with changing the strong views on the part of the emergency services staff that any patient outside a health centre required to be awarded a higher priority for transport. In some areas emergency services managers utilised the dedicated 'maternity' vehicles to fill gaps within their 
services. During the implementation there was a need for extensive discussion between midwives and emergency services personnel to give effect to the new transport strategy.

This intervention demonstrates the benefits that accrued from transporting women with complications during pregnancy and labour as quickly as possible to an appropriate facility, facilitating access to appropriate skills to manage the emergencies. The impact can therefore be attributed to addressing the second delay of Thaddeus and Maine. ${ }^{\left[{ }^{33}\right.}$ There was no change in issues relating to the first delay, as the BBA rate remained essentially unchanged (approximately 9\%). If the number of emergency vehicles cannot be increased nationally, then other interventions, such as the establishment of maternity waiting homes, should continue.

\section{Conclusions}

This study reflects a before-and-after analysis of data that were collected monthly from 2 separate databases. The correlation between maternal mortality and vehicle-dispatch data for maternity transfers indicates that the mortality improvements are clearly associated with improved inter-facility transfers.

This study outlines the impact of addressing the second-level delay on improving maternal outcomes by getting the patient to an appropriate facility in a reasonable time.

This analysis, reflecting one province's experience, provides strong evidence that health authorities should prioritise inter-hospital transport vehicles within their budgets, to ensure access of the pregnant population to appropriate care and, where feasible, to assign dedicated vehicles to maternity transfers.

Conflict of interest. This article was compiled from the routine data collected by the $\mathrm{DoH}$ on formal databases and does not contain any patient identifiers. Permission for publication was granted by the Free State DoH. The comments of Prof. Pattinson and Dr Moji are acknowledged. The author has no conflicts of interest to declare.

\section{References}

1. Fournier P, Du Mont A, Tourigny C, Dunkley G, Dramè S. Improved access to comprehensive obstetric care and its effect in institutional maternal mortality in rural Mali. Bull World Health Organ 2009;87:30-38. [http://dx.doi.org/10.2471/BLT.07.047076]

2. De Vries S, Wallis LA, Maritz D. A retrospective evaluation of the impact of a dedicated obstetric and neonatal transport service on transport times within an urban setting. Int J Emerg Med 2011;4(1):1-6. [http://dx.doi.org/10.1186/1865-1380-4-28]

3. Thaddeus S, Maine D. Too far to walk: maternal mortality in context. Soc Sci Med 1994;38(8):10911110. [http://dx.doi.org/10.1016/0277-9536(94)90226-7]

4. Saving Mothers 2008-2010 Short report. Pretoria: National Department of Health, 2012. http:// www.doh.gov.za/docs/reports/2012/Report_on_Confidential_Enquiries_into_Maternal_Deaths_in_ South_Africa.pdf (accessed 22 February 2013).

5. Rooks JP, Weatherby NL, Ernst EK, Stapleton S, Rosen D, Rosenfield A. Outcomes of care in birth centers. The National Birth Center Study. N Engl J Med 1989;321(26):1804-1811. [http://dx.doi org/10.1056/NEJM198912283212606

6. Murray SF, Pearson SC. Maternity referral systems in developing countries: current knowledge and future research needs. Soc Sci Med 2006;62(9):2205-2215. [http://dx.doi.org/10.1016/j. socscimed.2005.10.025

7. Schoon MG, Kabane S, Whitaker S. Maternity related adverse outcomes in the Free State province. Priorities at perinatal care conference Proceedings 2011. http://www.perinatalpriorities.co.za/database (accessed 22 February 2013)

Accepted 2 April 2013 